\title{
MODELING AND CONTROL OF THE IES PROJECT ROV
}

\author{
Rui M. F. Gomes, João B. Sousa and Fernando Lobo Pereira \\ Engineering Faculty of Porto University \\ \{rgomes,jtasso,flp\}@fe.up.pt \\ Rua Dr. Roberto Frias, s/n 4200-465 Porto PORTUGAL \\ Tel: +351225081539 Fax: +351225081443
}

Keywords: Remotely Operated Vehicles, Underwater Inspection, modeling of Underwater Vehicles, Sliding Modes.

\begin{abstract}
This paper describes the modeling and control of a Remotely Operate Vehicle (ROV), as part of the effort performed in the context of the Inspection of Underwater Structures (IES) project. The main objective of IES is the implementation of a ROVbased system for the inspection of underwater structures. The IES project is a 3 yearlong effort funded by the Portuguese R\&D program Praxis XXI. After addressing the complete model, including the body model and the thruster model, we present the design of a nonlinear controller based on the Sliding Modes methodology, which allow the vehicle to move according to the application requirements. PID controllers were also used in order to compare both performances.
\end{abstract}

\section{Introduction}

Although the market for inspection systems based on remotely operated vehicles is much more significant in the oil and gas industry than in any other, there has been an interesting growth in the use of such systems for other applications involving the inspection of underwater structures as well as other services. Examples of those include archeology, human and object salvage, power and communications cables deployment and maintenance, pipeline inspection, oceanographic research, de-mining, underwater civil works follow up, inspection of quays, bridges and dam walls, monitoring water tank and ship hull inspections.

The main goal of this paper concerns the design of control systems for underwater vehicles as part of inspection systems to address some of the mentioned applications. Therefore, in this work, we address issues concerning the modeling of underwater vehicles and, more specifically, a remotely operated vehicle (ROV) of the Underwater Systems and Technologies Laboratory (USTL). The modeling effort is organized into two main components: underwater rigid body motion and thrusters. All these components where integrated in a MATLAB simulation based environment which was instrumental as a framework to support control systems design.

The underwater vehicle motion, and, in particular, its positioning and trajectory tracking, pose a number of issues which are critical in the fulfillment of the requirements set out for the inspection activities. Since the model of the vehicle is nonlinear, a sliding modes controller was chosen to deal with the positioning of the inspection platform. A linear controller (PID) was also considered to fulfill the application requirements. The corresponding simulation results are presented and used in order to support a comparative analysis.

This paper is organized as follows. In section 2, we describe the IES project and the control problem. Section 3 presents the main parts of the non-linear ROV model. Section 4 describes the design of the control system and shows some simulations results. Finally, section 5 ends with some concluding remarks, and future work.

\section{The IES Project}

The project Inspection of Underwater Structures (IES) concerns the design and implementation of an advanced low cost system for the inspection of underwater structures based on a Remotely Operated Vehicle (ROV). The project started in 1999 with a duration of 3 years, and is funded by PROGRAMA PRAXIS XXI - MEDIDA 3.1B, Portugal. IES is a collaborative project that involves the Administração dos Portos de Douro e Leixões (APDL), Faculdade de Engenharia do Porto (FEUP) and Instituto de Sistemas e Robótica - Pólo do Porto (ISR).

The main innovations of the IES system with respect to commercially available ROV solutions are:

- On-board power and computer systems.

- Tele-programming operating mode. The tele-programming mode enables the operator to program automated inspection operations.

- Integrated navigation [10]. The IES navigation system integrates data provided by several types of sensors.

- Advanced control systems [6]. The ROV control system includes advanced automated operation modes.

- Open system. The project uses standard software development tools and principles.

Except for the ROV frame, hull and thrusters, all the other components and systems were designed and implemented at USTL. The ROV frame, hull and thrusters are a customized version of the Deep Ocean $500 \mathrm{~S}$ model from Deep Ocean Engineering. The main difference with respect to the standard model is an additional cylinder that houses electronics and sensors.

In the basic configuration the IES system comprises the following systems:

Computer system: Consists of a PC-104 stack running the real-time QNX operating system on the ROV, and a Windows 


\begin{tabular}{|l|l|}
\hline Manoeuvre & Description \\
\hline \hline Hovering & Maintain position and orientation \\
\hline GotoXY & Go from X to Y \\
\hline Orientation & Changes orientation \\
\hline Trajectory & Follow a desired trajectory \\
\hline Plane & Motion control constrained to a plane \\
\hline FollowWall & Follow a wall \\
\hline$\ldots$ & $\ldots$ \\
\hline
\end{tabular}

Table 1: Elemental Manoeuvres

based PC console connected through an Ethernet cable. The PC-104 computer system runs the command, control and navigation software. Basically, this computer accepts high-level commands from the console, and informs the console about the system state.

Power system: The umbilical cable feeds the ROV with two main power lines, one feeds 4 motors and 4 lights and the other, the electronics. Inside the ROV, there is a power conversion unit that generates all the necessary power levels. This design option aims at minimizing the effects of the tether on the ROV dynamics, one of the main difficulties associated with ROV operations.

Motor control system: This system comprises two CAN nodes housed in the two upper vehicle electronic cylinders. The controllers generate PWM reference signals to the four thruster power drives. DC motors are powered by these drivers.

Navigation system: The available sensors are: magnetic compass, inclinometers, inertial navigation unit, depth cell, altimeter, DVL and an acoustic system which is composed by two or more acoustic beacons. Sensor fusing based on Kalman filtering is used to determine the system state variables, which include three positions, three orientations, and their velocities.

Vision system: In the basic configuration, the vision system consists of a camera mounted on a pan-and-tilt unit and a spot light. The video image is converted to the digital format in the on-board frame-grabber and sent to the console through a ethernet connection to the console.

We organize the ROV operations as a sequence of manoeuvres. First, we define a basic set of "elemental manoeuvres", [4] from which all the manoeuvres can be derived. Once we have found a minimal set of elemental manoeuvres, we can verify their design for safety. We then compose the complex ROV manoeuvres, using the elemental manoeuvres as building blocks. Refer to table 1 . This form of defining the trajectory for the system can e achieved by a tri-level control architecture:

- Supervisory Layer - controls and coordinates the execution of manoeuvres.

- Manoeuvre Layer - control and observation subsystems are responsible for safe execution of manoeuvres - the first level of automation. The supervisory layer commands the execution of elemental manoeuvres according to some motion plan. Interactions with the regulation layer are mediated by the elemental manoeuvres. Each elementary ma- noeuvre sends low-level commands to the regulation layer and receives events concerning their completion or failure. Elemental manoeuvre control is given in terms of hybrid automata. The current design uses protocols in the form of finite state machines.

- Regulation Layer - The dynamical models of the vehicles are given in terms of non-linear ordinary differential equations. This level deals with continuous signals, and interfaces directly with the vehicle hardware. Control laws at this level correspond to low-level commands.

The control architecture shows the need of a control system for each elementary manoeuvre. Each manoeuvre will generate a trajectory, which must be executed by the regulation level. The regulation layer instantiates the correct controllers to accomplish the current manoeuvre. The greatest challenge in the manoeuvres execution is the design of efficient controllers for each manoeuvre. To design those controllers, it is important to know the system model. Therefore we will present the ROV model in the next section. In section 4 one of the several possible controllers will be designed in some detail to be implemented in the on-board ROV computer.

\section{The ROV Model}

The ROV model is composed of three main components: the rigid body, the propeller and the DC motor [3, 9, 12].

The rigid body model can be derived from the Newton-Euler formulation. The Newton-Euler formulation is based on Newton's Second Law in terms of conservation of both linear and angular momentum.

Another important issue when modeling 6 DOF (Degrees Of Freedom) systems is the specification of reference frames. It is important to define two main coordinate frames: the body-fixed and the earth-fixed. The body-fixed is attached to the vehicle. Its origin is normally fixed on the centre of gravity. The motion of the body-fixed reference frame is described relative to the earth-fixed reference frame. The earth-fixed reference frame can be considered inertial for low velocity vehicles such as the ROV.

Practical issues explain the need of two different reference frames. For example, it is easier to measure position and orientation on the earth-fixed reference frame instead in the body-fixed. The velocity is usually measured in the body-fixed reference frame. The notation defined by SNAME (Society of Naval Architects and Marine Engineers) is as follows:

Position and orientation (earth-fixed):

$$
\eta=\left(\eta_{1}, \eta_{2}\right)=(x, y, z, \phi, \theta, \psi)
$$

Linear and angular velocity (body-fixed):

$$
v=\left(\nu_{1}, \nu_{2}\right)=(u, v, w, p, q, r)
$$

There is also a kinetic transformation, which maps the transformation between both frames. This transformation is based on Euler angles

$$
\dot{\eta}=J\left(\eta_{2}\right) v
$$


where

$$
J\left(\eta_{2}\right)=\left[\begin{array}{cc}
J_{1}\left(\eta_{2}\right) & 0_{3 \times 3} \\
0_{3 \times 3} & J_{2}\left(\eta_{2}\right)
\end{array}\right]
$$

with

$$
\begin{gathered}
J_{1}\left(\eta_{2}\right)=\left[\begin{array}{ccc}
c \psi c \theta & -s \psi c \phi+c \psi s \theta s \phi & s \psi s \phi+c \psi c \phi s \theta \\
s \psi c \theta & c \psi c \phi+s \phi s \theta s \psi & -c \psi s \phi+s \theta s \psi c \phi \\
-s \theta & c \theta s \phi & c \theta c \phi
\end{array}\right] \\
J_{2}\left(\eta_{2}\right)=\left[\begin{array}{ccc}
1 & s \phi t \theta & c \phi t \theta \\
0 & c \theta & -s \phi \\
0 & s \phi / c \theta & c \phi / c \theta
\end{array}\right]
\end{gathered}
$$

and $s=\sin (),. c=\cos (),. t=\tan ($.$) .$

This transformation is undefined for $\theta= \pm 90^{\circ}$. To overcome this singularity, a quaternion approach must be considered. However, in the IES project this problem does not exist because the vehicle is not required to operate on $\theta= \pm 90^{\circ}$. Moreover, the vehicle is completely stable in roll and pitch, and the thruster actuation is not enough to force the vehicle to operate near those angles.

\subsection{Rigid body model}

The equations of motion can be written in the body-fixed frame as:

$$
\begin{gathered}
M \dot{v}+C(v) v+D(v) v+g(\eta)=\tau \\
\dot{\eta}=J\left(\eta_{2}\right) v
\end{gathered}
$$

where $v$ is the velocity of the vehicle in the body-fixed frame, $\eta$ the position and the orientation in the inertial frame, $M$ the inertia and added mass matrix, $C(v)$ the Coriolis and centripetal matrix, $D(v)$ the damping matrix, $g(\eta)$ the restoring forces and moments and $\tau$ the body-fixed forces from the actuators and other disturbances as currents and waves. Next, some details about the matrices, which compose equation (5), are presented.

The $\mathrm{M}$ matrix has two distinct contributions [2]. One is from the rigid body inertia. The other, appears when there is water motion and is called added mass. The added mass should be understood as pressure-induced forces and moments due to a forced harmonic motion of the body which are proportional to the acceleration of the body.

Some simplifications were made in this matrix. The origin of the body-fixed reference frame was chosen to be the gravity centre. It is very difficult to get all the 36 added mass parameters, and therefore we use only the diagonal $6 \times 6$ matrix with the diagonal entries $m-X_{\dot{u}}, m-Y_{\dot{v}}, m-Z_{\dot{w}}, I_{x x}-K_{\dot{p}}$, $I_{y y}-M_{\dot{q}}, I_{z z}-N_{\dot{r}}$, where $m$ is the vehicles mass, $I_{x x} . . I_{z z}$ are the inertia moment and $X_{\dot{u}} . . N_{\dot{r}}$ are the added mass coefficients.

The $\mathrm{C}$ matrix represents the effect of the Coriolis and centripetal forces in the vehicle. Like the previous matrix, the $\mathrm{C}$ matrix also has two distinct contributions, one from the rigid body and other from the added mass. The $\mathrm{C}$ matrix is repre- sented in equation (7)

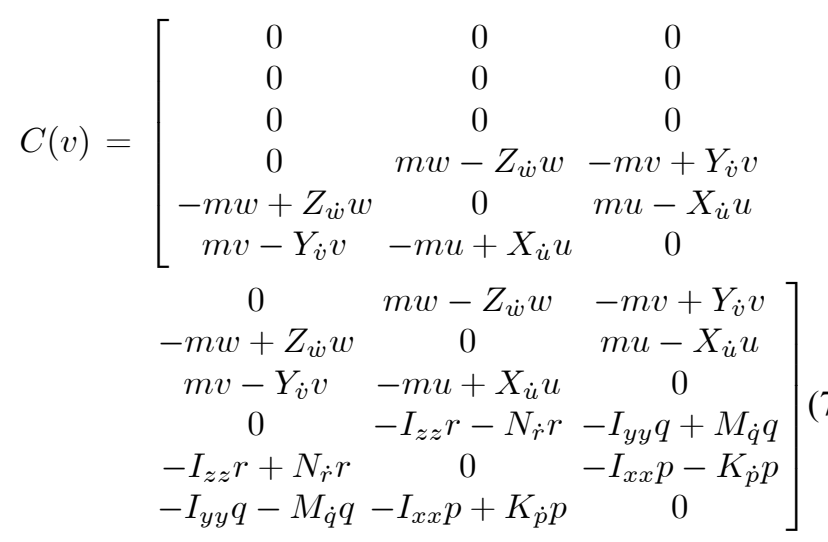

D matrix represents the hydrodynamic damping. Potential damping, wave drift damping, skin friction and vortex shedding damping cause the main damping. Usually, the damping of underwater vehicles moving in 6 DOF is highly non-linear and coupled. Nevertheless, if the system has three planes of symmetry and we assume it is decoupled, then D matrix becomes diagonal: $D=\operatorname{diag}\left(-X_{u|u|}|u|-X_{u},-Y_{v|v|}|v|-Y_{v},-Z_{w|w|}|w|-Z_{w}\right.$, $\left.-K_{p|p|}|p|-K_{p},-M_{q|q|}|q|-M_{q},-N_{r|r|}|r|-N_{r}\right)$.

$g$ vector encompasses the effects of the restoring forces and moments, namely the gravitational and the buoyancy ones. Assuming that the vehicle is neutral in the water, the restoring forces and moments become

$$
g(\eta)=\left[\begin{array}{c}
0 \\
0 \\
0 \\
-B G_{y} W c \theta c \phi+B G_{z} W c \theta s \phi \\
B G_{z} W s \theta+B G_{x} W c \theta c \phi \\
-B G_{x} W c \theta s \phi-B G_{y} W s \theta
\end{array}\right]
$$

where $W$ is the gravity force, $B$ the buoyancy force and $B G_{i}$ the distance between the gravity centre and the buoyancy centre in all $i$ directions $x, y$ and $z$.

The $\tau$ vector (9) are the thrusters forces and moments, which are applied to the vehicle. In our ROV system, we have only four thrusters installed. This will be seen again in the section 4 . Two of them are positioned in the rear part of the vehicle and thrusts it in the forward way. Another thruster, forces the vehicle to move up and down. And the last one moves the vehicle sideway.

$$
\tau=\left[\begin{array}{c}
F_{1}+F_{2} \\
F_{3} \\
F_{4} \\
-F_{3} D_{3 z}+F_{4} D_{4 y} \\
F_{1} D_{1 z}+F_{2} D_{2 z}-F_{4} D_{4 x} \\
-F_{1} D_{1 y}-F_{2} D_{2 y}+F_{3} D_{3 x}
\end{array}\right]
$$

$F_{1} F_{2} F_{3}$ and $F_{4}$ are the forces produced by the rear port thruster, the rear starboard thruster, the lateral thruster and vertical thruster respectively. $D_{i j}$ is the distance between the force application point and the gravity centre in the $\mathrm{j}$ direction. 


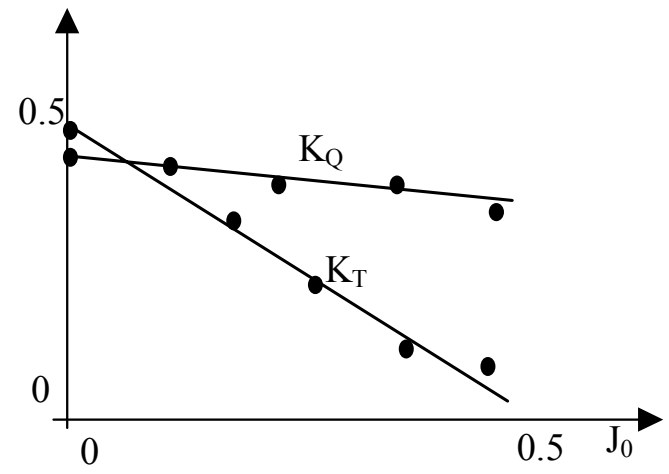

Figure 1: Propeller parameters

\subsection{Propeller model}

A thruster is composed by a motor (in our case a DC motor) and a propeller $[3,8]$. The motor equations are very well known and therefore we skip it presentation. The propeller model is a bit difficult to achieve. Some of the difficulties are:

- the separation of the drag forces of the vehicle and the propeller thrust;

- complex hydrodynamic behaviors like vortex shedding on the propeller blades, ummodeled blades, duct effects.

The forces and moments produced by the propeller depend on both the vehicle and the propeller velocities. A first order model approximation is usually used

$$
\begin{aligned}
& T=\rho D^{4} K_{T}\left(J_{0}\right)|n| n \\
& Q=\rho D^{5} K_{T}\left(J_{0}\right)|n| n
\end{aligned}
$$

where $\rho$ is the water density, $D$ the propeller diameter, $n$ the propeller revolution, $K_{T}\left(J_{0}\right)$ the propeller coefficient and $J_{0}$ the advance number. The advance number is given by

$$
J_{0}=\frac{V_{A}}{n D}
$$

where $V_{A}$ is the advance speed.

The advance speed is the speed of the water going into the propeller. To model a thruster is necessary to make some tests on a pool. The target is to collect several $\left(J_{0}, T\right)$ and $\left(J_{0}, Q\right)$ pairs. These pairs can be converted in $\left(J_{0}, K_{T}\right)$ and $\left(J_{0}, K_{Q}\right)$ pairs with the following expression.

$$
K_{T}=\frac{T}{\rho D^{4}|n| n} \quad K_{Q}=\frac{Q}{\rho D^{5}|n| n}
$$

Then those pairs can be represented in a graphic, like the one presented in figure 1 . Once we have a large number of pairs, it is possible to get a linear regression which leads to

$$
\begin{aligned}
& K_{T}=\alpha_{1}+\alpha_{2} J_{0} \\
& K_{Q}=\beta_{1}+\beta_{2} J_{0}
\end{aligned}
$$

The final forces and moments are given by

$$
\begin{aligned}
& T=\rho D^{4}\left(\alpha_{1}+\alpha_{2} J_{0}\right)|n| n \\
& Q=\rho D^{5}\left(\beta_{1}+\beta_{2} J_{0}\right)|n| n .
\end{aligned}
$$

\section{Control Design}

The control problem is to choose the actuation forces to be applied so that the vehicle reaches the desired inertial coordinates $[1,3,11]$.

In order to fulfill the requirements of the elemental manoeuvres control problem, a PID based and multivariable Sliding Modes controllers were designed.

The use of a single linear controller leads to an inconsistent performance that varies with the velocity of the system. Linear techniques can stabilize nonlinear systems such as underwater vehicles but it is necessary to design a set of linear controllers to achieve good performances. Each of them must be designed for a different system velocity. As system velocity varies with time, the current linear controller is replaced by another that handles the control task with advantage. This approach is not very suitable for a vehicle that moves in several directions because there are no obvious operating points to linearize the system. A possible solution could be to consider a very large number of linear controllers designed for combinations of all axis velocities. Ideally, the exact model without any linearization should be considered in the control design. The sliding modes approach fills this requirements $[7,13,14]$. This controller is a particular case of Variable Structure Control System (VSCS). A VSCS is characterized by a set of control laws and a decision rule. The decision rule, also known as switching function measures the system behavior and informs which controller must be chosen in that instant. A VSCS can be seen as a set of subsystems where each subsystem is a control law and can be used in a region of the state space. The sliding mode methodology encompasses two main steps. The design of a switching function that satisfies the specifications and the design of a control law that forces the switching function to be attractive for the systems state.

Since the vehicle is under-actuated, we had to consider a 4 DOF model to derive the corresponding control law. Although the controller is based on the 4 DOF model, it is used to control the complete model (6 DOF). We do this because we know that the system is stable in both roll and pitch variables.

Assuming a 4 DOF ROV model in the body-fixed reference frame like the one presented on the equation (5), and considering a multivariable sliding mode controller [3], it was defined:

- a Lyapunov function of the form

$$
V(s, t)=\frac{1}{2} s^{T} M s \quad, \quad M=M^{T}>0,
$$

- a sliding surface like

$$
s=\dot{\tilde{x}}+\lambda \tilde{x}
$$

where $\tilde{x}=x-x_{d}$ is the system error.

- a virtual reference $x_{r}$ such that

$$
s=\dot{x}-\dot{x}_{r} \quad \text { where } \quad \dot{x}_{r}=\dot{x}_{d}-\lambda \tilde{x}
$$

- a virtual reference $q_{r}$ (fixed reference frame) such that

$$
\dot{x}_{r}=J(x) \dot{q}_{r} .
$$


This yield the following control law:

$$
\begin{aligned}
u= & B^{-1}(\underbrace{\hat{M} \ddot{q}_{r}+\hat{C} \dot{q}_{r}+\hat{D} \dot{q}_{r}+\hat{g}}_{\text {feedforward term }}-\underbrace{J^{T} K_{D} s}_{\mathrm{PD}} \\
& -\underbrace{K \times \operatorname{sgn}\left(J^{-1} s\right)}_{\text {Robustifying term }})
\end{aligned}
$$

where

$$
\begin{aligned}
& \dot{q}_{r}=J^{-1} \dot{x}_{r} \\
& \ddot{q}_{r}=J^{-1}\left(\ddot{x}_{r}-\dot{J} J^{-1} \dot{x}_{r}\right)
\end{aligned}
$$

In order to get $\dot{V} \leq 0$ it is sufficient that

$k_{i} \geq\left|\hat{M} \ddot{q}_{r}+\tilde{C}(\dot{q}) \dot{q}_{r}+\tilde{D}(\dot{q}) \dot{q}_{r}+\tilde{g}(x)\right|_{i}+l_{i}, \quad l_{i}>0$

where $\tilde{M}=\hat{M}-M, \tilde{C}=\hat{C}-C, \tilde{D}=\hat{D}-D$ and $\tilde{g}=\hat{g}-g$. This is known as the sliding condition.

In order to validate the control system, we propose some simulations. The inputs of the control system are the following:

$$
\begin{aligned}
\eta_{d} & =\left[\begin{array}{llll}
x_{d} & y_{d} & z_{d} & \psi_{d}
\end{array}\right] \\
v_{d} & =\left[\begin{array}{llll}
u_{d} & v_{d} & w_{d} & r_{d}
\end{array}\right] \\
a_{d} & =\left[\begin{array}{llll}
\ddot{x}_{d} & \ddot{y}_{d} & \ddot{z}_{d} & \ddot{\psi}_{d}
\end{array}\right] \text { (inertial) }
\end{aligned}
$$

We assume that there is a trajectory generation system [5] which gives us these desirable values and that there are several sensors to measure the vehicle position and velocity

$$
\begin{aligned}
& \eta=\left[\begin{array}{llll}
x & y & z & \psi
\end{array}\right] \\
& v=\left[\begin{array}{llll}
u & v & w & r
\end{array}\right]
\end{aligned}
$$

The outputs are obviously all four thrusters. Let us assume that we want to stabilize the vehicle in the origin $(0,0,0,0)$ starting with the initial conditions $(2,2,2, \pi / 2)$. We performed two different simulations. One based on nonlinear control (Sliding modes) and the other on PID. The results of this simulation are presented in figure 2 (Sliding Modes in solid line and PID in dashed line). As we can see, all the controlled variables go to 0 as time goes to $\infty$, but due to the coupled motion of the vehicle, the PID controller can not stabilize the y variable in acceptable time. Moreover, if we change to a different kind of motion we need to tune the PID parameters again. This means that the PID is trying to control an important nonlinear model assuming that it is linear. Therefore the Sliding modes controller is much more suitable than the PID one.

Another important issue is the vehicle actuation. As we can see in figure 3 the actuators get saturated a little bit in both cases at the beginning of the simulation (@130V). Although this saturation is very small, when it happens the control system become open loop, which is not very good. Even in this situation, the vehicle is controlled to the desired position. This scenario can be worse if we add model parameters uncertainty to the feedback control system. Another simulation was addressed in order to verify this effect. The parameters that are not very well known in our model are: $I_{z z}, X_{\dot{u}}, Y_{\dot{v}}, Z_{\dot{w}}, N_{\dot{r}}$, $X_{u|u|}, Y_{v|v|}, Z_{w|w|}, N_{r|r|}, X_{u}, Y_{v}, Z_{w}$ and $N_{r}$ (refer to section
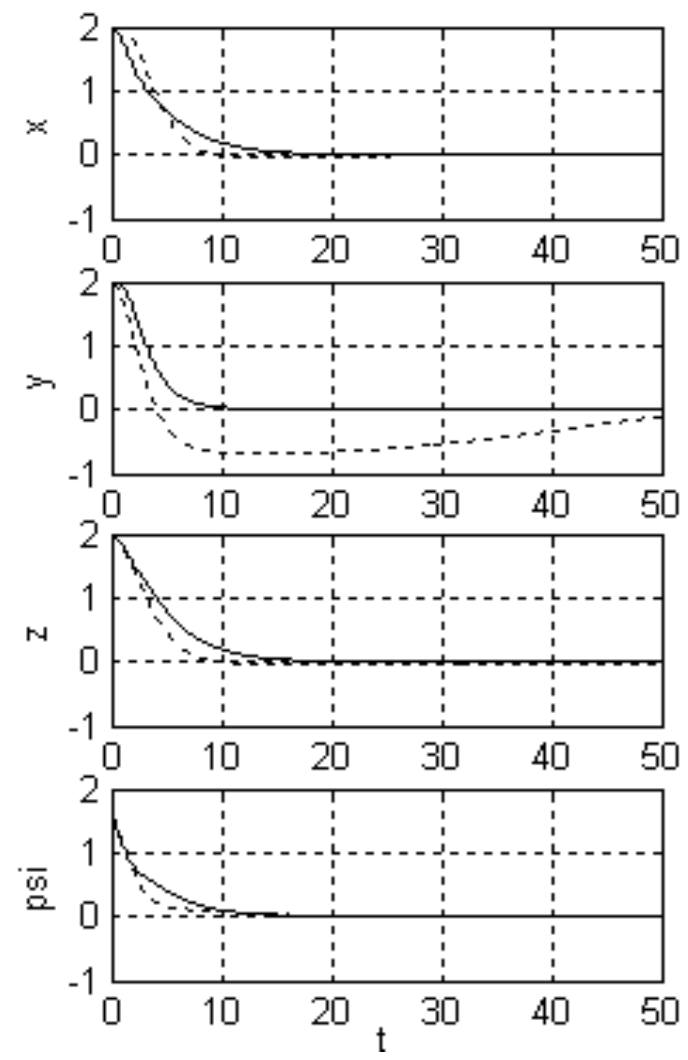

Figure 2: Sliding modes and PID controllers comparison

3). These are also the most influents in the motion proposed for this simulation. The model parameters were allowed to be modified from $-50 \%$ to $+50 \%$ the nominal value. The chosen value was $+50 \%$ because if we rise those parameters, the ROV platform becomes slower and the controller output will rise to accomplish the mission. By looking to the figure 4, it can be seen that the output become saturated for a longer period of time than in the first situation (compare 2 with 1).

\section{Conclusions and Future Work}

In this paper was presented the mathematical model of the IES ROV. The equations presented models the ROV in terms of rigid body, propeller and DC motor. Simulations done previously, show that this model is not very accurate, but it is also known that is very difficult to get a good model for this kind of systems. To obtain a good model it would be necessary to take the vehicle to a test tank (with PMM systems) to get more accurate parameters.

To compensate this, it was used a robust controller - sliding modes. A simulation was addressed to compare the performances of both the PID and sliding modes controllers. Obviously the results obtained by the sliding modes are much better than the other. At our laboratory, other simulations regarding the robustness of the controller were performed and proved to be a very good controller for the manoeuvres proposed at the beginning of this paper.

In the future, we will test the controller to disturbances like 

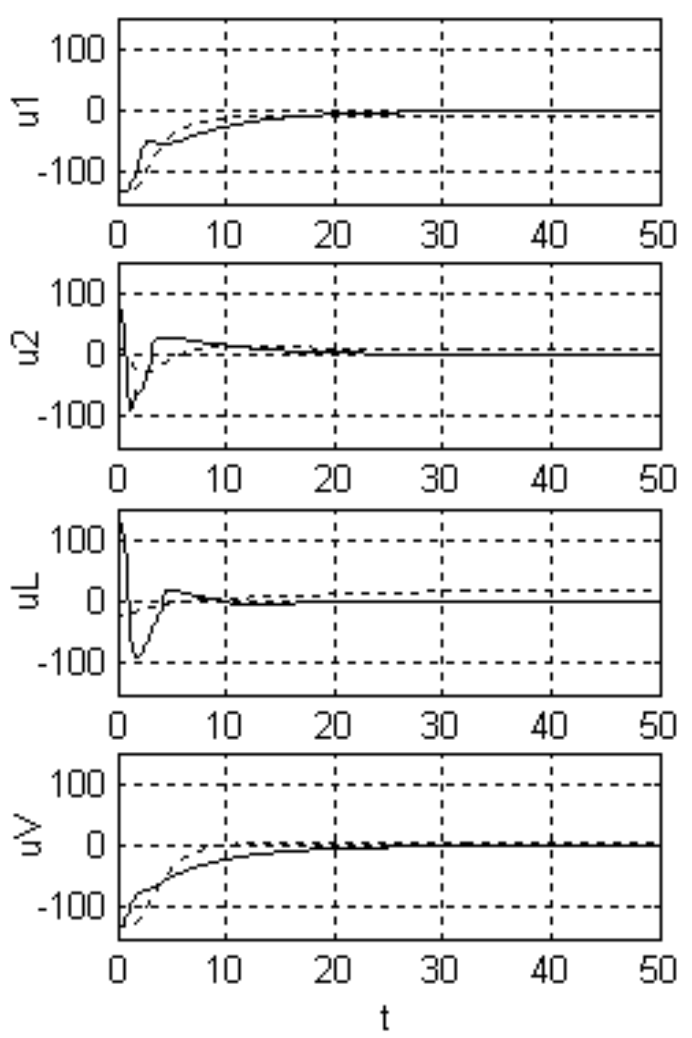

Figure 3: Vehicle actuation

currents, waves and the umbilical tether force. Another important issue is to compare the performance of this controller with others like, state feedback linearization or adaptive, in order to determine which one is more appropriate to each manoeuvre.

To improve the performance of inspection activities, a controller for the Pan\&Tilt unit supporting a video camera will be designed. Since the response of this system is much faster than that of the ROV platform, its control can compensate with advantage the effect of disturbances that were not eliminated by the platform control system.

\section{Acknowledgments}

This material is based upon work funded by the PRAXIS XXI - Medida 3.1b (IES project) and by Ministério da Defesa, Portugal. I want to thank the Portuguese Science and Technology Foundation (FCT) for the finance support.

\section{References}

[1] J. Borges de Sousa, F. Lobo Pereira, and E. Pereira Silva. A dynamically configurable control architecture for autonomous mobile robots. Proceedings of the 34th IEEE Conference on Decision and Control, 1995.

[2] Thor I. Fossen. Guidance and Control of Ocean Vehicles. John Whiley and Sons, 1995.

[3] Thor Inge Fossen. Nonlinear Modelling and Control of Underwater Vehicles (PHD). 1991.

[4] Sérgio L. Fraga, João B. Sousa, Anouck Girard, and Al-
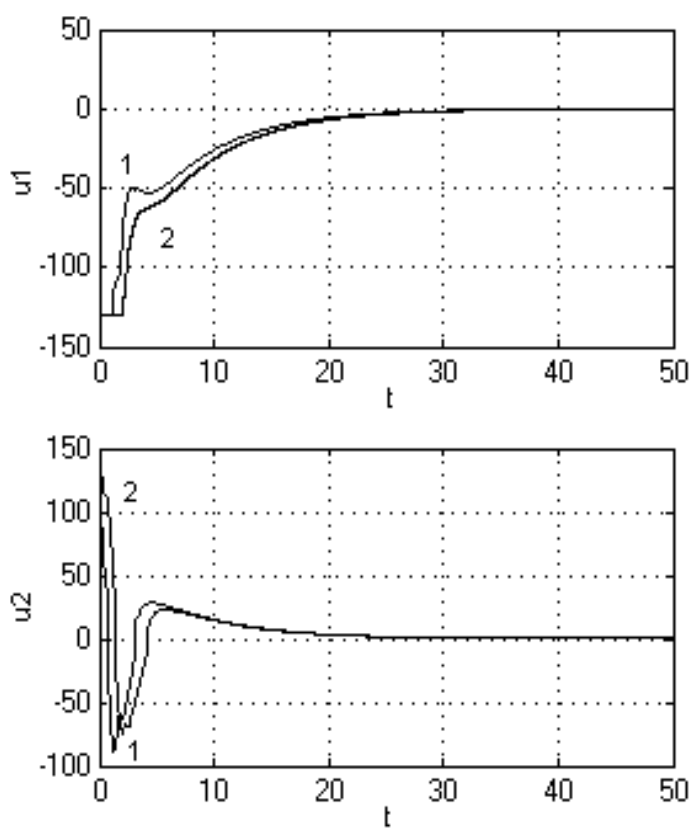

Figure 4: Comparison of controls in the forward motion. (1) Nominal parameters $(2)+50 \%$ on the nominal parameters

fredo Martins. An automated maneuver control framework for a remotely operated vehicle. Oceans, 2001.

[5] Sérgio Loureiro Fraga. Geração de Trajectórias - Aplicação a Veículos Subaquáticos (MSC). FEUP, 2002.

[6] Rui Manuel Ferreira Gomes. Controlo e Modelização de Veículos Subaquáticos (MSC). FEUP, 2002.

[7] A. Healey and D. Lienard. Multivariable sliding mode control for autonomous diving and steering of unmanned underwater vehicles. IEEE J. Ocean Eng., pages 327339, 1993.

[8] D. Yoerger L. Whitcomb. Development, comparison, and preliminary experimental validation of nonlinear dynamic thruster models. IEEE Journal of Oceanic Engineering, Vol. 24, 1999.

[9] E. Lewis. Principles of Naval Architecture. Society of NavalArchitects and Marine Engineers, 1989.

[10] A. Matos, A. Martins, N. Cruz, and F. Pereira. Development and implementation of a low-cost LBL navigation system for an AUV. MTS/IEEE Oceans '99, Seattle, U.S.A, 1999.

[11] Richard M. Murray, Zexiang Li, and S. Shankar Sastry. A Mathematical Introduction to Robotic Manipulation. CRC Press, 1994.

[12] J. N. Newman. Marine Hydrodynamics. MIT Press, 1977.

[13] Dana R. Yoerger, James B. Newman, and Jean-Jacques E. Slotine. Supervisory control system for the JASON ROV. IEEE J.Oceanic Eng., pages 392-399, 1986.

[14] Dana R. Yoerger and J. J. E. Slotine. Robust trajectory control of underwater vehícles. IEEE J. Ocean Eng., 1985. 